\section{Floral Product Behaviors and Their Influence on Consumer Floral Purchase Frequency}

\author{
Li-Chun Huang ${ }^{1}$
}

\begin{abstract}
ADDITIONAL INDEX WORDs. flower, floral market, factor analysis,
\end{abstract} multinomial logistic regression

\begin{abstract}
SumMary. Product behavior represents how consumers perceive and use a product. Its importance in predicting consumer buying behavior is well documented in marketing research. There are, however, no data available investigating the role of product behavior in the floral market. This study addressed this deficiency. Data were first analyzed using factor analysis to extract the principal determinants of product behavior in the floral market. As a result, six primary behavioral factors were identified and named as: "using flowers as daily essentials," "perceived product value," "negative attitude toward flowers," "using flowers as gifts," "eventbased usage," and "experience in receiving flowers." The effects of these extracted behavioral factors on consumer flower purchase frequency were then further investigated with multinomial logistic regression analysis. Analytical results revealed that behaviors "using flowers as daily essentials" and "using flowers as gifts" forced consumers to become heavy users in the floral market. Conversely, "negative attitude toward flowers" negatively affected the floral purchase frequency. Experimental results in this study also suggest that promoting a positive attitude toward flowers is essential in encouraging consumers to become flower users. The intended use of flower product purchase, whether for personal use or as gifts, was the main factor affecting the frequent purchasing of flowers.
\end{abstract}

$\mathrm{P}$ roduct behavior comprises consumer behavior patterns, which reveal how consumers use or respond to a product. Product behavior is typically measured by consumer product knowledge, attitude, loyalty, and product benefits sought. As such, understanding consumer product behavior helps marketers develop efficient marketing strategies. Due to the capability of product behavior to differentiate between consumers, product behavior variables are often employed as instruments for market segmentation [Kotler, 2003 (p. 278-306); Miller and Granzin, 1979; Tynan and Drayton, 1987].

Product behavior in numerous markets has been well studied. However, there are no product behavior studies for the floral market. Although

${ }^{1}$ DaYeh University, Taiwan, R.O.C.; e-mail address: lc24h@mail.dyu.edu.tw

The author would like to thank Dr. Chao-Min Hsu for his valuable discussions. Thanks are also due to Dr. Charles Hall and the anonymous reviewers for their review and insightful comments on an earlier version of this manuscript. previous research in the floral market does not focus on product behavior, it does present cues about how consumers perceive floral products. For instance, in a psychographics study, Demby (1973) found that various consumer groups had different behaviors toward floral products, such as product attitudes and occasions for using flowers. Some consumers perceived flowers as expensive, while others viewed flowers as daily essentials, and felt no guilt in buying flowers for themselves (Demby, 1973). In terms of occasions for flower usage, Demby found that some consumers used flowers daily, while others only used flowers for special occasions, such as for holidays, funerals, or when visiting the ill. Intended use influenced consumer floral purchase decision; the purpose of personal use was especially effective for floral market segmentation (Behe et al., 1992a, 1992b).

Demby (1973) also noted that consumers sought a variety of product benefits from flowers; that is, flowers could be purchased for personal purposes, or to facilitate and enhance social relationships.

How consumers behave toward flowers is culturally dependent. In a Japanese study of flower appreciation, Matsuo (1990) found that in addition to the visual appeal of flowers, Japanese consumers also considered growing flowers as a process of fostering life; that is, flowers also fulfill spiritual requirements for Japanese people.

Some research also provides cues regarding the potential effect of product behavior on flower purchasing. Demby determined that floral purchase frequency and quantity were related to consumer perceptions and attitudes about flowers. In a marketing segmentation study, Behe and Wolnick (1991a) also found that consumers who purchased more flowers exhibited a higher level of floral knowledge. Furthermore, most of these consumers purchased flowers for themselves, rather than as gifts. However, amount of floral knowledge did not influence consumer purchasing behavior for flower choice (Behe and Wolnick, 1991b). Still, knowledge about how product behavior functions in the floral market is very limited. This study addresses this deficiency.

Product behavior has been measured traditionally with the variables of consumer knowledge, attitude, use, or response to a product. But the applicability of these variables to the floral market has not been identified. Thus, identifying the main factors of product behavior in the floral market will allow researchers and marketers to predict the effects of product behavior on floral purchase frequency.

Floral purchase frequency is a measure of the maturation of a floral market. In a poorly developed floral market, floral purchase frequency is about 1-2 times per year on a per capita basis. If the floral market is fully developed, per-person purchases will be approximately 15-20 times per year (De Boon, 1990). Since floral purchase frequency is important in measuring the degree of floral market development and its potential relationship with consumer floral product behaviors (Demby, 1973), this study examines the hypothesis that floral product behavior significantly influences consumer floral purchase frequency, and thereby explores the importance of consumer floral product behavior in the floral market. 
Factor analysis was first used to extract the main factors of product behavior in the floral market. Next, multinomial logistic regression analysis was performed and the significance and effect of each factor on consumer floral purchase frequency were evaluated. The results of this study can provide researchers and marketers with an understanding of significant floral product behaviors in the floral market, thus assisting them in increasing flower sales.

There are two objectives in this study: 1) to define the main factors of consumer product behavior in the floral market; and 2) to evaluate the impact of these factors on consumer floral purchase frequency.

\section{Materials and methods}

QuestionnAIre Design. A 29question survey was developed to evaluate consumer product behavior for flowers. The questions, both openended and close-ended, were informed by the results of several previous studies (Behe and Wolnick, 1991a, 1991b; Behe et al., 1992a, 1992b; Demby, 1973; Lee, 1996; Matsuo, 1990). Thus, the validity of these questions was protected. The questions addressed consumer knowledge and use of flowers, as well as price perception, quality perception, purchase motivation, and the benefits required of flowers. Responses were measured on a five-point Likert scale $(5=$ extremely agree, $4=$ agree, $3=$ neutral, $2=$ disagree, and $\mathrm{l}=$ extremely disagree).

Purchase frequency was measured by asking consumers how many times on average they purchase flowers in a year. Socioeconomic data for the study participants were also collected.

SAMPling. Consumers from six main urban administrative districts participated in this study: Yancheng, Sanmin, Sinsing, Cianjin, Lingya, and Cianjhen, in Kaohsiung, Taiwan. Kaohsiung is the second largest city in Taiwan, and the primary center of numerous industries and business. The main sectors of the Kaohsiung economy are goods and services, agriculture, forestry, fishing, and animal husbandry. Kaohsiung is also home to the largest commercial harbor in Taiwan; its cargo volume ranks third in the world. There is also an international airport located in Kaohsing for passengers from all over the world. Kaohsiung has more than 20 international sister cities. Many in- ternational activities on foreign culture, arts, and business are held annually in this city. Due to its diverse lifestyles and international status, Kaohsiung was chosen as the survey location to improve the global generalizabilty of this study's outcomes.

Convenient sampling was performed at public sites, such as department stores, train stations, parks, markets, etc. After agreeing to participate, participants were asked to complete the questionnaire on floral products. In this study, floral products were defined as fresh products of cut flowers, potted flowering plants, or foliage plants. Most of the participants took approximately 10 to $15 \mathrm{~min}$ to complete the questionnaire. The survey was conducted between 7 and 10 Oct. 2001, not associated with any event. A total of 378 consumers participated in this study: 200 participants were recruited from the districts of Lingya, Sanmin, and Yancheng; and 178 were surveyed from Sinsing, Cianjin, and Cianjhen. Of the total returned questionnaires, 62 were incomplete and excluded from the study. Therefore, 316 questionnaires were used for statistical analysis.

Statistical analysis. The participants' floral product behavior was analyzed with factor analysis to extract the main factors for floral product behavior. Floral purchase frequency was then transformed into a nominal scale, and a multinomial logistic regression analysis was utilized to analyze how these behavior factors influenced participant floral purchase frequency. The multinomial logistic regression method is a modified logistic regression analysis that can handle the case situations in which the dependent variable is nominal and has more than two levels. All statistical analyses were processed with SPSS (version 10.0; SPSS, Inc., Chicago).

\section{Results and discussion}

Participants were aged between 18 and 63 years; $67.8 \%$ were female and $32.2 \%$ were male. Participant occupations were as follows: manufacturing $(5.4 \%)$; commercial business $(15.7 \%)$; civil servant $(4.2 \%)$; education $(4.5 \%)$; military service (5.1\%); students $(24.4 \%)$; service industry $(25.6 \%)$; managers $(4.8 \%)$; housekeeping (4.2\%); and medical care $(0.6 \%)$. A total of $5.4 \%$ of participants did not indicate their occupation. A significant number of participants
(24.0\%) reported that they had a monthly income of (New Taiwanese dollar) NT $\$ 30,000$ or less; $23.7 \%$ had an income between NT\$30,001 and NT\$60,000; and $19.9 \%$ had an income over NT\$60,000. Almost one-third (32.5\%) of participants did not provide their income information.

MAIN FACTORS OF PRODUCT BEHAVIOR IN THE FLORAL MARKET. Responses were first analyzed with factor analysis to extract the main behavior factors for the floral market with factorability examined before the factor analysis was processed. The correlation matrix of the data revealed that 311 of the 406 correlations $(76.6 \%)$ were significant at the level of $\alpha=0.05$. This finding proved that it was adequate to examine factorability with the Bartlett test and the measure of sampling adequacy (MSA). Bartlett's test of sphericity was significant at the level of $\alpha=0.001\left(\chi^{2}=2981.63\right)$, and the overall MSA value was 0.89 . Both results demonstrate the suitability of applying factor analysis to this data set [Hair et al., 1998 (p. 100)]. Since the MSA values for each variable were larger than 0.50 , all 29 variables were suitable for factor analysis [Hair et al., 1998 (p. 99-100)].

Factor analysis was processed with principal component analysis and Varimax rotation. The extraction criteria were 1) eigenvalue $>1$, and 2) factor loading $\geq 0.50$ [Hair et al., 1998 (p. 105, 111)]. Of the 29 variables for floral product behavior, nine variables were deleted. As a result, six factors were extracted as the main dimensions of product behavior for the floral market (Table 1).

The first factor, "using flowers as daily essentials," was extracted from the following component variables: "flowers increase the quality of life," "I read books or magazines that talk about flowers," "flowers are essential for daily life," "I buy flowers for home decoration," "I buy flowers to increase the beauty of daily life," and "I know the names of many or some flowers." These variables indicated a tendency to buy flowers daily, to see flowers as essential to daily life, and consumer familiarity with flowers. The analysis of rotation sums of squared loadings in factor analysis showed that this factor accounted for $16.84 \%$ of the total variance in participants' floral product behavior.

The second factor was extracted 
from thefollowingvariables: "sometimes the flowers I purchase are not fresh enough," "the flowers I purchase do not last long," "flaws are usually found on the flowers I purchase," "flowers are expensive," and "most flowers are hard to take care of." These component variables primarily referred to perceptions of price and quality. This factor was therefore named "perceived product value" to denote the total product value perceived by participants. Based on the analytical results, consumers evaluated the value offlowers from numerous perspectives, such as monetary value, quality, longevity, etc. This factor accounted for $11.30 \%$ of the total variance in participant floral product behaviors.

The third factor was extracted from the following variables: "flowers are not a practical product," "flowers are just plants, they mean nothing to me," and "flowers are a luxury." This factor was suitably named "negative attitude toward flowers." Participants with these attitudes viewed flowers as useless or impractical, and buying flowers as a waste of money. This factor accounted for $10.78 \%$ of the total variance in participant floral product behaviors.
The fourth factor was extracted from the following variables: "I buy flowers as a wedding gift for my friends," "when visiting relatives or friends, I purchase flowers as a gift," and "flowers are an ideal gift." These three component variables primarily indicated that participants purchase flowers as gifts. This factor, which accounted for $9.57 \%$ of the total variance, was named "using flowers as gifts."

The fifth factor was extracted from the following variables: "I buy flowers for special occasions," and "I buy flowers for religious worship." This factor was named "event-based usage" to signify that flowers were used on special occasions, rather than daily. This factor accounted for $6.58 \%$ of the total behavioral variance.

The sixth, the final factor, was based on a single variable: "someone has given me flowers before." This factor was aptly named "experience in receiving flowers."

With the analytical results, variables for consumer floral product behavior can be summarized into the primary factors as follows: using flowers as daily essentials; perceived product value; negative attitude to- ward flowers; using flowers as gifts; event-based usage; and experience in receiving flowers. These six factors accounted for $61.58 \%$ of the total variance in consumer floral product behavior.

For subsequent multinomial logistic regression analysis, factor scores were generated with regression to become the measurements of these extracted behavioral factors.

THE IMPACT OF FLORAL PRODUCT BEHAVIOR ON CONSUMER FLORAL PURCHASE FREQUENCY. Multinomial logistic regression analysis was applied to evaluate the impact of floral product behavior on floral purchase frequency. With the principles of multinomial logistic regression analysis, logits of the odds ratios between two outcome categories of a dependent variable are in a linear function to the independent variables. Assume there are $p$ number of independent variables $\left(x_{p} \mathrm{~s}\right), P(\mathrm{y}=$ $i$ ) is the conditional probability of the dependent outcome in the outcome category $i$, and $P(\mathrm{y}=j)$ is the conditional probability in another outcome category $j$; then the logit of $P(y=j)$ vs. $P(\mathrm{y}=i)$ is in a linear function to the independent variables $x_{p}$ s. Generally, if there are $n$ outcome categories in

Table 1. Summary of factor analysis performed to identify the main factors of participant floral product behavior and the component variables of the main factors identified, based on 316 valid questionnaires surveyed in Kaohsiung, Taiwan, between 7 and 10 Oct. 2001.

\begin{tabular}{|c|c|c|c|}
\hline Component variables $^{\mathrm{z}}$ & $\begin{array}{c}\text { Factor } \\
\text { loadings }\end{array}$ & $\begin{array}{c}\text { Validity } \\
(\text { Cronbach } \alpha)\end{array}$ & Main factors ${ }^{\mathrm{x}}$ \\
\hline $\begin{array}{l}\text { Flowers increase the quality of life. } \\
\text { I read books or magazines that talk about flowers. } \\
\text { Flowers are essential for daily life. } \\
\text { I buy flowers for home decoration. } \\
\text { I buy flowers to increase the beauty of daily life. } \\
\text { I know the names of many or some flowers. }\end{array}$ & $\begin{array}{l}0.774 \\
0.750 \\
0.691 \\
0.686 \\
0.666 \\
0.624\end{array}$ & 0.835 & Using flowers as daily essentials \\
\hline $\begin{array}{l}\text { Flowers are not a practical product. } \\
\text { Flowers are just plants; they mean nothing to me. } \\
\text { Flowers are a luxury. }\end{array}$ & $\begin{array}{l}0.799 \\
0.779 \\
0.629\end{array}$ & 0.733 & Negative attitude toward flowers \\
\hline $\begin{array}{l}\text { I buy flowers for special occasions. } \\
\text { I buy flowers for religious worship. }\end{array}$ & $\begin{array}{l}0.843 \\
0.644\end{array}$ & 0.430 & Event-based usage \\
\hline Someone has given me flowers before. & 0.772 & --- & Experience in receiving flowers \\
\hline
\end{tabular}

${ }^{2}$ Measured with participants' responses on a five-point Likert scale $(5=$ extremely agree, $4=$ agree, $3=$ neutral, $2=$ disagree, and $\mathrm{l}=$ extremely disagree $)$.

'Rotation method was varimax with Kaiser normalization, converged in eight iterations.

${ }^{x}$ Extraction method was principal component analysis. 
the dependent variable, there will be a total of $(n-1)$ logits in the multinomial regression model. These logit functions, expressed as $\mathrm{g}_{\mathrm{j}}(x)$, can be summarized as a multinomial logistic regression equation:

$$
\begin{aligned}
\mathrm{g}_{\mathrm{j}}(x)= & \ln \left[\frac{P(y=j)}{P(y=i)}\right]=\beta_{j 0}+\beta_{j 1} X_{1} \\
& +\beta_{j 2} X_{2}+\beta_{j 3} X_{3}+\ldots+\beta_{j p} X_{p}
\end{aligned}
$$

where $\beta$ s are the parameters for interpreting the ratio of the probability of outcome category $j[P(\mathrm{y}=j)]$ to that of the baseline category $[P(\mathrm{y}=i)]$. In multinomial logistic regression analysis, one of the outcome categories is usually chosen as the baseline category whose probability is used as the comparison baseline for each other outcome category when constructing the logit equation set in a multinomial logistic regression model. By solving the coefficients of the independent variables in the logit equations, the effect of each independent variable on a dependent variable is evaluated and compared. In a multinomial logistic regression model, coefficients for the independent variables are estimated with a maximum likelihood function. These coefficients allow for interpretation of the odds of each outcome category in a dependent variable. Furthermore, once any independent variable changes in amount or at nominal level, the exponentiation of the estimated $\mathrm{co}^{-}$ efficients also functions in predicting the amount of change in odds ratios of outcome categories vs. the baseline category [Hosmer and Lemeshow, 2000 (p. 264-273)].

Participant floral purchase frequency was originally measured on a numeric scale with an open-ended question to which participants were free to record their number of annual floral purchases. To apply multinomial logistic regression analysis, data of floral purchase frequency were transformed into four levels of categorical data: zero purchase, low-frequency purchase, medium-frequency purchase, and high-frequency purchase. Zero purchase signifies no purchase of flowers ( $18.0 \%$ of participants). Low-frequency purchase represents a purchasing frequency of $1-2$ times per year $(37.9 \%)$. Medium-frequency purchase represents a purchasing frequency of 3-14 times per year (39.4\%). High-frequency purchase represents a purchasing frequency of 15 or more times per year $(4.7 \%)$. These category boundaries were determined with De Boon's proposition regarding the development of a floral market (1990), which states that floral purchase frequency is an indicator of a floral market's maturity: in an undeveloped floral market, the purchase frequency is 1-2 times per year; and when a floral market is well developed, purchase frequency increases to at least 15 times per year.

As the dependent variable of floral purchase frequency was transformed into a nominal variable with four outcome categories, there were a total of three logit functions in the multinomial logistic regression model. The probability of a zero purchase was used as the baseline to form logits; therefore, these three logits are:

$$
\begin{aligned}
& \mathrm{g}_{1}(x)=\ln \left[\frac{P(y=1 \sim 2)}{P(y=0)}\right], \\
& \mathrm{g}_{2}(x)=\ln \left[\frac{P(y=3 \sim 14)}{P(y=0)}\right], \\
& \text { and } \mathrm{g}_{3}(x)=\ln \left[\frac{P(y \geq 15)}{P(y=0)}\right],
\end{aligned}
$$

where $P(y=0), P(y=1 \sim 2), P(y=3 \sim 14)$, and $P(y \geq 15)$ refer to, respectively, the probabilities of the four different outcome categories: zero purchase, low-frequency purchase, mediumfrequency purchase, high-frequency purchase. When the extracted factors of participant floral product behavior served as independent variables, the logit functions in this study were summarized as a multinomial logistic regression equation:

$$
\begin{aligned}
\mathrm{g}_{\mathrm{j}}(x)= & \ln \left[\frac{P(y=j)}{P(y=0)}\right]=\beta_{j 0}+\beta_{j 1} X_{1} \\
& +\beta_{j 2} X_{2}+\beta_{j 3} X_{3}+\ldots+\beta_{j p} X_{p}
\end{aligned}
$$

The extracted factors of participant floral product behavior were adopted as the independent variables $X_{p} \mathrm{~s}$, to interpret the ratio of the probability of outcome category $j[P(y=j)]$ vs. that of the baseline category zero purchase $[P(y=0)]$, where $\beta_{j p}$ is the parameter for the relative independent variable $X_{p}$.

Backward variable elimination was used in this study to build the best-fit multinomial logistic regression model. The significance of variables was assessed via the likelihood ratio chisquare test. Consequently, behavioral variables "perceived product value" $\left(\chi^{2}=5.426, P=0.143\right)$, "event-based usage" $\left(\chi^{2}=3.729, P=0.292\right)$, and "experience in receiving flowers" $\left(\chi^{2}=\right.$ $7.224, P=0.065)$ were eliminated at the level of $\alpha=0.05$. Behavioral variables "using flowers as daily essentials" $\left(\chi^{2}=\right.$ 29.764, $P=0.000)$, "negative attitude toward flowers" $\left(\chi^{2}=10.491, P=\right.$ $0.015)$ and "using flowers as gifts" $\left(\chi^{2}\right.$ $=22.923, P=0.000)$ were identified as significant independent variables in model building $(\alpha=0.05)$. Table 2 presents the final statistical results. From the estimated coefficients in the multinomial logistic regression model, the following two problems were solved: 1) the functional relationship between floral product behavior and floral purchase frequency; and 2) the quantitative effect of floral product behavior on floral purchase frequency. These two concerns were addressed for each significant factor.

"USING FLOWERS AS DAILY ESSENTIALS." The behavior factor "using flowers as daily essentials" was significant in the logistic function of participant flower purchase frequency. The exponentiation of estimated $\mathrm{co}^{-}$ efficients (category of zero purchase as the baseline) identified that with a one-unit increase (as measured on the five-point Likert scale) participants would be 1.935 times more likely to purchase flowers at low frequency (odds ratio, 1.935), 3.236 times more likely to purchase flowers at a medium frequency (odds ratio, 3.236), and 3.891 times more likely to purchase flowers at high frequency (odds ratio, 3.891). Obviously, participants who consider flowers as essential to daily life buy flowers more frequently than those who do not consider flowers as essentials.

"Negative attitude toward FLOWERs." Multinomial logistic regression analysis showed that behavior factor "negative attitude toward flowers" had a negative effect on participant floral purchase frequency. As participants' attitudes became more negative by one point on the Likert scale, the probability ratio of low-frequency for floral purchase vs. zero purchase decreased with the ratio of 0.484 ; decreased at the ratio of 0.456 for medium-frequency purchasing vs. zero purchase. The factor "negative attitude toward flowers" had an obvious and negative impact on participant floral purchase frequency. The more negative 
Table 2. Summary of multinomial logistic regression analysis performed to evaluate the effect of floral product behavior on consumer floral purchase frequency, based on 316 valid questionnaires surveyed in Kaohsiung, Taiwan, between 7 and 10 Oct. 2001.

\begin{tabular}{|c|c|c|c|c|c|c|c|}
\hline \multirow{3}{*}{$\begin{array}{l}\text { Floral product } \\
\text { behavior }^{z, y}\end{array}$} & \multirow{2}{*}{$\begin{array}{c}\begin{array}{c}\text { Category of } \\
\text { purchase frequency }^{x}\end{array} \\
\text { High-frequency purchase }\end{array}$} & \multirow{2}{*}{$\frac{\boldsymbol{\beta}^{\mathrm{w}}}{1.359}$} & \multirow{2}{*}{$\frac{\text { SE }}{0.439}$} & \multirow{2}{*}{$\frac{P}{0.002}$} & \multirow{2}{*}{$\begin{array}{l}\begin{array}{l}\text { Odds } \\
\text { ratio }\end{array} \\
3.891^{*}\end{array}$} & \multicolumn{2}{|c|}{$\begin{array}{l}95 \% \text { Confidence } \\
\text { interval for odds ratio }\end{array}$} \\
\hline & & & & & & 1.644 & 9.206 \\
\hline & Medium-frequency purchase & 1.174 & 0.245 & 0.000 & $3.236^{*}$ & 2.003 & 5.229 \\
\hline & Low-frequency purchase & 0.660 & 0.207 & 0.001 & $1.935^{*}$ & 1.290 & 2.902 \\
\hline & Zero purchase & -- & --- & --- & 1 & --- & -- \\
\hline \multirow{4}{*}{$\begin{array}{l}\text { Negative attitude } \\
\text { toward flowers }\end{array}$} & High-frequency purchase & -0.599 & 0.375 & 0.110 & 0.549 & 0.263 & 1.146 \\
\hline & Medium-frequency purchase & -0.786 & 0.233 & 0.001 & $0.456^{*}$ & 0.289 & 0.719 \\
\hline & Low-frequency purchase & -0.726 & 0.218 & 0.001 & $0.484^{*}$ & 0.316 & 0.742 \\
\hline & Zero purchase & --- & --- & --- & $\mathrm{l}$ & --- & --- \\
\hline \multirow{4}{*}{ Using flowers as gifts } & High-frequency purchase & 1.014 & 0.384 & 0.008 & $2.758^{\star}$ & 1.299 & 5.856 \\
\hline & Medium-frequency purchase & 0.953 & 0.231 & 0.000 & $2.592^{*}$ & 1.647 & 4.081 \\
\hline & Low-frequency purchase & 0.424 & 0.210 & 0.044 & $1.527^{\star}$ & 1.011 & 2.307 \\
\hline & Zero purchase & --- & --- & --- & 1 & --- & --- \\
\hline
\end{tabular}

${ }^{\mathrm{x}}$ Main factors of participant floral product behavior extracted from factor analysis as presented in Table 1.

'Measured on the factor scores generated from the computing function associated with the performance of factor analysis presented in Table 1 .

'Categorized with participants' annual average floral purchase frequency: zero purchase represents no purchase of flowers; low-frequency purchase represents a purchasing frequency of 1-2 times per year; medium-frequency purchase represents a purchasing frequency of 3-14 times per year; and high-frequency purchase represents a purchasing frequency of 15 or more times per year.

wEstimated parameters for interpreting the ratio of the probability of a outcome category to that of the baseline category, for the dependent variable of floral purchase frequency.

"Zero purchase served as the baseline category.

*Significant at $\alpha=0.05$.

the attitudes of participants toward flowers, the less likely they may be to use flowers. However, this influence was nonsignificant for high-frequency purchasing vs. zero purchase.

"Using FlOWERS AS GIFTS." The behavior factor "using flowers as gifts" also significantly influenced participant floral purchase frequency. With a oneunit increase in the tendency to use flowers as a gift, participants would be 1.527 times more likely to purchase flowers at low frequency (odds ratio, 1.527), 2.592 times more likely to purchase flowers at medium frequency (odds ratio, 2.592), and 2.758 times more likely to purchase flowers at high frequency (odds ratio, 2.758), when compared with zero purchase. Thus, for those who are more inclined to buy flowers as gifts, the more likely that they will become frequent floral purchasers.

\section{Conclusion}

Although consumers have different preferences and attitudes regarding flowers, this study found that floral behavior variables are interrelated and can be condensed into a simple set of variables. Floral behavior variables tested with factor analysis in this study were condensed into six primary behavior factors. Analytical results indicated that the measurement of consumer product behavior in the floral market can be simplified and measured by these six dimensions rather than the entire set of variables. According to the analysis of rotation sums of squared loadings in factor analysis, $61.58 \%$ of the total variance in participant floral product behavior can be explained with these six behavior factors.

Product behavior has been identified as critical in forecasting purchasing behavior. How important are these extracted factors in predicting consumer flower buying behavior? Multinomial logistic regression analysis found that the behavioral factors "using flowers as daily essentials," "negative attitude toward flowers," and "using flowers as gifts" were significant in affecting participant floral purchase frequency. When participants are more likely to use flowers daily or use flowers as gifts, they are more likely to become flower users, and especially to become heavy flower users who purchase flowers much more frequently than those who do not have such floral behaviors. A negative attitude toward flowers had a negative influence on flower purchase frequency. When participants have a negative attitude toward flowers, they tend to become flower non-users who very rarely purchase flowers. However, a negative attitude was nonsignificant for high-frequency purchase. The intended use of flowers was obviously the main variable that encourages consumer motivation to buy flowers frequently, regardless of whether the flowers are for personal use or gifts. This finding is similar to that obtained by Baourakis et al. (2000). In research of the EU floral market, Baourakis et al. indicated that a higher income is not the primary variable for flower purchasing; that is, an increased number of floral purchases mainly depended on the intended use of the floral products. Therefore, to encourage consumers to become heavy flower users, marketing programs should aim at creating positive attitudes about flowers, and create more reasons and occasions that motivate flower purchasing.

Although the factors "perceived product value," "event-based usage," and "experience in receiving flowers" are nonsignificant for participant floral purchase frequency, this does not mean that they are unimportant to the floral market. This study was limited by its statistical data, which used floral purchase frequency as the only dependent variable in exploring the importance of floral product behavior in the floral market. Potential relations between floral product behavior and other important variables in the floral market remain unexplored. For a comprehensive understanding of the role of product behavior in the floral market, future research should focus on how product behavior influences 
consumer preference for floral products, their selection of retail channels, and their purchase decision mode in the floral market.

\section{Literature cited}

Baourakis, G., D. Gerasopoulos, N. Kalofolias, N. Kalogeras, and A. Zoumis. 2000. Marketing research: The case of floral products. Acta Hort. 541:227-232.

Behe, B.K., T.A. Prince, and H.K. Tayama. 1992a. Analysis of consumer purchases of floral products in supermarkets. HortScience 27(5):455-459.

Behe, B.K., T.A. Prince, and H.K. Tayama. 1992b. Market segmentation of supermarket floral customers. HortScience 27(5):459-462.

Behe, B.K. and D.J. Wolnick. 199la. Market segmentation of Pennsylvania floral consumers by purchase volume and primary retail outlet. HortScience 26(10):1328-1331.

Behe, B.K. and D.J. Wolnick. 1991b. Type of floral product purchased and demographic characteristics and floral knowledge of consumers. HortScience 26(4):414-416.

De Boon, I.H. 1990. A world perspective on more flowers for more people. Professional Plant Growers Assn. News 21:7,10-11.
Demby, E. 1973. A psychographic study of the market for flowers. Amer. Florists Mktg. Council, Alexandria, Va.

Hair, J.F., Jr., R.E. Anderson, R.L. Tatham, and W.C. Black. 1998. Multivariate data analysis, intl. ed., 5th ed. Prentice-Hall, Upper Saddle River, N.J.

Hosmer, D.W. and S. Lemeshow. 2000. Applied logistic regression, 2nd ed. Wiley, New York.

Kotler, P. 2003. Marketing management, llth ed. Prentice-Hall, Upper Saddle River, N.J.

Lee, H. 1996. An analysis of household expenditure for flowers (in Chinese). J. Agr. For. 45(4):21-28.

Matsuo, E. 1990. Analysis of flower appreciation and its international comparison contribute to progress of flower production and international flower trade. HortScience 25:1468-1471.

Miller, K.E. and K.L. Granzin. 1979. Simultaneous loyalty and benefit segmentation of retail store customers. J. Retailing 55(1):47-60.

Tynan, A.C. and J. Drayton. 1987. Market segmentation. J. Mktg. Mgt. $2(3): 301-335$. 